\title{
Chiral Symmetry Breaking with the Curtis-Pennington Vertex
}

\author{
D. Atkinson, V.P. Gusynin' and P. Maris \\ Institute for Theoretical Physics \\ Nijenborgh 4 \\ 9747 AG Groningen, The Netherlands \\ E-mail: ATKINSON@TH.RUG.NL
}

\begin{abstract}
We study chiral symmetry breaking in quenched $\mathrm{QED}_{4}$, using a vertex Ansatz recently proposed by Curtis and Pennington. Bifurcation analysis is employed to establish the existence of a critical coupling and to estimate its value. The main results are in qualitative agreement with the ladder approximation, the numerical changes being minor.
\end{abstract}

\footnotetext{
${ }^{1}$ Permanent address: Institute for Theoretical Physics, Ukrainian Academy of Sciences, 252143 Kiev, Ukraine.
} 


\section{Chapter 1}

\section{Introduction}

The study of chiral symmetry breaking in quantum field theory requires the use of non-perturbative techniques. A commonly used method is based on solving the Dyson-Schwinger equations in the so-called rainbow or ladder approximation, which uses a bare vertex [1], [2], [3], 四]. However, such a truncation of the infinite set of integral equations in QED does not in general respect two fundamental requirements: the Ward-Takahashi identity and multiplicative renormalizability. This shortcoming calls in question all the results of the ladder approximation.

Recently Curtis and Pennington [5] presented a very interesting Ansatz for the full three-point vertex that satisfies the Ward-Takahashi identity and which is multiplicatively renormalizable to all orders in leading and nextto-leading logarithms in perturbation theory. They showed how powerful the constraints on the form of the vertex are. The natural question is to what extent the Curtis-Pennington modification to the ladder approximation alters the physical results, in particular the value of the critical coupling for onset of chiral symmetry breaking. Applying bifurcation theory to the Curtis-Pennington equations, we find that there is indeed a non-zero critical coupling; moreover the numerical changes as compared with the ladder approximation will be shown to be fairly small.

These relatively minor numerical modifications encourage the hope that the essential results of the ladder approximation survive vertex modifications. 
However, caution is necessary in espousing the point of view that the full theory has the same properties, since it is known [6] that vacuum polarization effects do modify the nature of the chiral-symmetry phase transition. Indeed, mean field behaviour appears to supervene. Nevertheless, the mutual influence of two essentially distinct but related effects, that of charge screening and of 'fall into the centre', have not yet been consistently taken into account. The basic question: 'Does the ladder approximation already contain the essence of the physics of chiral symmetry breaking?' is therefore still open.

In this paper we shall neglect all vacuum polarization effects and we restrict attention to the Landau gauge. Moreover we neglect another known deficiency of the ladder approximation, namely the occurrence of spurious first-sheet complex singularities in the fermion propagator [7]. We write the equations from the beginning in Euclidean space and postpone the question of their relevance to Lorentz space. 


\section{Chapter 2}

\section{Curtis-Pennington Equations}

In Euclidean space our notation for the unrenormalized fermion propagator ist

$$
S_{F}(p)=\frac{A^{-1}\left(p^{2}\right)}{\gamma p+m\left(p^{2}\right)} .
$$

The Landau gauge bare photon propagator has the form

$$
D_{F}^{\mu \nu}(k)=\frac{1}{k^{2}}\left(\delta^{\mu \nu}-\frac{k^{\mu} k^{\nu}}{k^{2}}\right),
$$

and the Curtis-Pennington equations are

$$
A(x)=1-\frac{\alpha_{0}}{4 \pi} \int_{0}^{\Lambda^{2}} \frac{d y}{y+m^{2}(y)} I(y, x)
$$

and

$$
A(x) m(x)=\frac{\alpha_{0}}{4 \pi} \int_{0}^{\Lambda^{2}} \frac{d y}{y+m^{2}(y)} J(y, x) .
$$

The functions $I$ and $J$, which depend explicitly on $m$ and $A$, were given in Ref. [5]. If we set $m(x)=0$, we find that $I$ and $J$ both vanish, in fact $I$ is of order $m^{2}$ while $J$ is of order $m$. Hence Eq.(2.3) and Eq.(2.4) possess the trivial solution $A(x)=1$ and $m(x)=0$. This is the only solution for small values of the coupling, $\alpha_{0}$.

\footnotetext{
${ }^{1}$ Curtis and Pennington's $\Sigma$ is our $m$ and their $F$ is our $A^{-1}$.
} 
In order to examine the possibility that a nontrivial solution, $m(x)$, branches away from the trivial one at a critical coupling, $\alpha_{c}$, we examine the so-called bifurcation equation 8]. This involves differentiating Eq.(2.3) and Eq.(2.4) functionally with respect to $m$ and then setting $m=0$. Since $I$ is secondorder in $m^{2}$, we find to first order in $\delta m$ that $I(y, x)=0$ and, from the Curtis-Pennington expressions,

$$
\begin{aligned}
J(y, x)= & 3 \delta m(y)\left[\frac{y}{x} \theta(x-y)+\theta(y-x)\right] \\
& -\frac{3 x}{2} \frac{\delta m(y)-\delta m(x)}{y-x}\left[\frac{y^{2}}{x^{2}} \theta(x-y)+\theta(y-x)\right]
\end{aligned}
$$

According to the standard rules of bifurcation theory, we should substitute $y$ for the denominator $y+m^{2}(y)$ in Eq.(2.4). However, this introduces a spurious infra-red singularity, which can be eliminated by replacing the denominator by $y+m^{2}$, where the constant $m$ can be fixed a posteriori by the normalization requirement

$$
\delta m(0)=m .
$$

We shall call $m$ the dynamical mass. Rescaling $x / m^{2} \longrightarrow x, y / m^{2} \longrightarrow y$, and $\delta m(x) / m \longrightarrow f(x)$, we obtain the basic bifurcation equation

$$
\begin{aligned}
f(x)= & \frac{\lambda}{x} \int_{0}^{x} \frac{y d y}{y+1}\left[f(y)-\frac{y}{2} \frac{f(y)-f(x)}{y-x}\right] \\
& +\lambda \int_{x}^{\frac{\Lambda^{2}}{m^{2}}} \frac{d y}{y+1}\left[f(y)-\frac{x}{2} \frac{f(y)-f(x)}{y-x}\right],
\end{aligned}
$$

where

$$
\lambda=\frac{3 \alpha_{0}}{4 \pi} .
$$

In the Landau gauge, the terms involving $\frac{f(y)-f(x)}{y-x}$ are all that is left of the complicated Curtis-Pennington Ansatz in the linearized form appropriate to a bifurcation analysis. 


\section{Chapter 3}

\section{Critical Coupling}

In this section we shall consider three approximate methods for handling the bifurcation equation (2.7):

1. $\frac{f(y)-f(x)}{y-x} \Longrightarrow 0$

2. $\frac{f(y)-f(x)}{y-x} \Longrightarrow f^{\prime}(y)$

3. $\frac{f(y)-f(x)}{y-x} \Longrightarrow f^{\prime}(\max (x, y))$.

\subsection{Second-order Equation}

Here we simply ignore the extra Curtis-Pennington term. As is well-known, the integral equation

$$
f(x)=\frac{\lambda}{x} \int_{0}^{x} \frac{y d y}{y+1} f(y)+\lambda \int_{x}^{\frac{\Lambda^{2}}{m^{2}}} \frac{d y}{y+1} f(y),
$$

then reduces to the standard hypergeometric differential equation

$$
x(x+1) f^{\prime \prime}+2(x+1) f^{\prime}+\lambda f=0,
$$


with infra-red and ultra-violet boundary conditions. The required solution is

$$
f(x)={ }_{2} F_{1}\left(\alpha_{1}, \alpha_{2} ; \gamma ;-x\right),
$$

where

$$
\alpha_{1}, \alpha_{2}=\frac{1}{2} \pm \sqrt{\lambda_{c}-\lambda} ; \quad \gamma=2 ; \quad \lambda_{c}=\frac{1}{4} .
$$

This solution of the differential equation is the only one that is finite at $x=0$ : the normalization is fixed by the infra-red condition $f(0)=1$, which corresponds to Eq.(2.6). From the integral equation we still have to impose the ultra-violet condition, $B\left(\frac{\Lambda^{2}}{m^{2}}\right)=0$, where

$$
B(x)=\frac{d}{d x}[x f(x)]={ }_{2} F_{1}\left(\alpha_{1}, \alpha_{2} ; 1 ;-x\right) .
$$

For $\lambda<\lambda_{c}, \alpha_{1}$ and $\alpha_{2}$ are real, and under these conditions the hypergeometric function Eq. (3.5) has no finite zeros: the ultra-violet condition cannot be satisfied for any $m \neq 0$, so there is no nontrivial solution of the integral equation. The imposition of the ultra-violet cut-off, $\Lambda$, is essential for this result [1], [2], [9], [10. From the physical point of view, it is easy to satisfy the axial-vector current conservation with a finite cut-off by just setting the bare mass equal to zero. Without an ultra-violet cut-off, one needs a more subtle treatment, distinguishing between an irregular solution, which exists for any coupling strength, but which leads to non-conservation of the axial-vector current, and the regular solution, which exists only if the coupling is large enough. It is not easy in a computer calculation to see the difference between these two types of solution unless one explicitly works with a cut-off off is also necessary in order to make sure that the bifurcation equation is of classical Fredholm type, so that the standard theory can be applied [8].

When $\lambda>\lambda_{c}, \alpha_{1}$ and $\alpha_{2}$ are complex conjugate numbers and the hypergeometric function Eq.(3.5), which remains real, has an infinite number of oscillations; the smallest zero, say $x_{0}$, can be chosen to define $m$ through the relation

$$
x_{0}=\frac{\Lambda^{2}}{m^{2}} .
$$

\footnotetext{
${ }^{1}$ In the second paper of Ref.5 Curtis and Pennington had claimed that a dynamical mass is generated for all values of the coupling. In fact they failed to make the above distinction between a regular and an irregular solution.
} 
For large $\Lambda$, the dynamical mass is given by

$$
m=\Lambda \exp \left[-\frac{\pi+\delta(\lambda)}{2 \sqrt{\lambda-\lambda_{c}}}\right]
$$

with

$$
\delta(\lambda)=\arg \frac{\Gamma^{2}\left(\frac{1}{2}+i \sqrt{\lambda-\lambda_{c}}\right)}{\Gamma\left(1+2 i \sqrt{\lambda-\lambda_{c}}\right)} .
$$

Near the critical point, $\lambda=\lambda_{c}=0.25$, Eq.(3.6) takes the form

$$
m=\Lambda \exp \left[-\frac{\pi / 2}{\sqrt{\lambda-\lambda_{c}}}+2 \log 2\right],
$$

whereas the mass function (3.3) behaves at large momenta as

$$
f(x) \sim \frac{1}{\sqrt{x}} \log x .
$$

The formulas (3.6) and (3.7) have played a great role in attracting attention to the non-perturbative study of QED. Firstly the critical point $\alpha_{c}=\frac{\pi}{3}$ has been interpreted [2, 3] as a nontrivial ultra-violet fixed point defining the continuum limit of QED. In fact, if we start from the supercritical phase and let $\Lambda \rightarrow \infty$, then in order to keep the dynamical mass finite we have to perform the charge renormalization as follows

$$
\alpha(\Lambda)=\alpha_{c}\left(1+\frac{\pi^{2}}{\log ^{2} \frac{4 \Lambda}{m}}\right) \longrightarrow \alpha_{c}=\frac{\pi}{3},
$$

as $\Lambda \rightarrow \infty$. Furthermore, the $\beta$-function of the theory,

$$
\beta(\alpha) \equiv \Lambda \frac{\partial \alpha}{\partial \Lambda}=-\frac{2}{3}\left(\frac{\alpha}{\alpha_{c}}-1\right)^{\frac{3}{2}}, \quad \alpha>\alpha_{c}
$$

has an ultra-violet stable zero at $\alpha=\alpha_{c}$. The existence of such a critical point was confirmed recently by computer simulations of noncompact QED on the lattice 11. Secondly, as one can see from Eq.(3.9), the fermion dynamical mass falls off with increasing momentum as $\frac{1}{p}$, and this is in sharp contrast to asymptotically free theories like QCD where $m\left(p^{2}\right) \sim \frac{1}{p^{2}}$ [12]. It was 
suggested [13] that this more slowly decreasing behaviour of the mass-function can solve the notorious flavour-changing neutral current problem in extended technicolour theories.

By proceeding beyond the ladder approximation, we would like to find out whether the results (3.6) and (3.7) are still valid or not. In Ref. 14, 15] evidence was adduced in favour of the validity of an equation of the type (3.6) at any finite order in the quenched approximation, and we shall make use of some of the arguments in Sect. 4. Our main concern here will be to take into account the vertex corrections in a manner compatible with the requirements of both the Ward-Takahashi identity and of multiplicative renormalizability.

\subsection{Third-order Equation}

With the second approximation, the integral equation reads

$$
\begin{aligned}
f(x)= & \frac{\lambda}{x} \int_{0}^{x} \frac{y d y}{y+1}\left[f(y)-\frac{y}{2} f^{\prime}(y)\right] \\
& +\lambda \int_{x}^{\frac{\Lambda^{2}}{m^{2}}} \frac{d y}{y+1}\left[f(y)-\frac{x}{2} f^{\prime}(y)\right],
\end{aligned}
$$

We shall show that this integral equation is equivalent to a third-order differential equation of generalized hypergeometric type, together with an infra-red and two ultra-violet boundary conditions.

A first differentiation of Eq.(3.10) gives

$$
\begin{aligned}
f^{\prime}(x)= & -\frac{\lambda}{x^{2}} \int_{0}^{x} \frac{y d y}{y+1}\left[f(y)-\frac{y}{2} f^{\prime}(y)\right] \\
& -\frac{\lambda}{2} \int_{x}^{\frac{\Lambda^{2}}{m^{2}}} \frac{d y}{y+1} f^{\prime}(y),
\end{aligned}
$$

which implies the infra-red condition

$$
\left.x^{2} f^{\prime}(x)\right|_{x=0}=0
$$


On combining Eq.(3.10) and Eq.(3.11) we find

$$
[x f(x)]^{\prime}=\lambda \int_{x}^{\frac{\Lambda^{2}}{m^{2}}} \frac{d y}{y+1}\left[f(y)-x f^{\prime}(y)\right] .
$$

A second differentiation gives

$$
[x f(x)]^{\prime \prime}=\frac{\lambda}{x+1}\left[x f^{\prime}(x)-f(x)\right]-\lambda \int_{x}^{\frac{\Lambda^{2}}{m^{2}}} \frac{d y}{y+1} f^{\prime}(y),
$$

and a third differentiation finally yields

$$
f^{\prime \prime \prime}+\left[\frac{3}{x}-\frac{\lambda}{x+1}\right] f^{\prime \prime}-\frac{\lambda}{x(x+1)^{2}} f^{\prime}-\frac{\lambda}{x(x+1)^{2}} f=0 .
$$

The first ultra-violet condition (from Eq.(3.13)),

$$
\left.\{x f(x)\}^{\prime}\right|_{x=\frac{\Lambda^{2}}{m^{2}}}=0,
$$

is as in the second-order case; but Eq.(3.14) gives rise to a new ultra-violet condition,

$$
\left.\left\{[x f(x)]^{\prime \prime}+\lambda \frac{f(x)-x f^{\prime}(x)}{x+1}\right\}\right|_{x=\frac{\Lambda^{2}}{m^{2}}}=0 .
$$

The above differential equation belongs to the class of equations of Fuchsian type with three regular singularities at the points $x=-1,0, \infty$. Moreover, changing the variable to $z=x+1$ and writing Eq.(3.15) as

$$
z^{2}(1-z) f^{\prime \prime \prime}+[-\lambda-(3-\lambda) z] z f^{\prime \prime}+\lambda f^{\prime}+\lambda f=0,
$$

we recognize it as the equation for the hypergeometric function, ${ }_{3} F_{2}$.

We choose as a linearly independent set of solutions

$$
f_{i}(z)=\left\{\prod_{\nu=1}^{3} \frac{\Gamma\left(\alpha_{i}+\gamma_{\nu}\right)}{\Gamma\left(\alpha_{i}-\alpha_{\nu}+1\right)}\right\} z^{-\alpha_{i}}{ }_{3} F_{2}\left(\begin{array}{l|l}
\alpha_{i}+\gamma_{1}, \alpha_{i}+\gamma_{2}, \alpha_{i}+\gamma_{3} \\
\alpha_{i}-\alpha_{1}+1, \alpha_{i}-\alpha_{2}+1, \alpha_{i}-\alpha_{3}+1
\end{array} \mid \frac{1}{z}\right)
$$

with the convention that the term $\alpha_{i}-\alpha_{j}+1$ in ${ }_{3} F_{2}$ is omitted when $i=j$. Here the $\alpha_{i}$ are the roots of the characteristic equation

$$
\alpha^{3}+\lambda \alpha^{2}+(\lambda-1) \alpha+\lambda=0
$$


and $\gamma_{1}=0, \gamma_{2}=1+\lambda, \gamma_{3}=2$. The $\alpha$ 's (which should not be confused with the coupling, $\alpha_{0}$ !) evidently satisfy

$\alpha_{1}+\alpha_{2}+\alpha_{3}=-\lambda, \quad \alpha_{1} \alpha_{2}+\alpha_{2} \alpha_{3}+\alpha_{3} \alpha_{1}=\lambda-1, \quad \alpha_{1} \alpha_{2} \alpha_{3}=-\lambda$.

The $\Gamma$ factors are included for later convenience.

The general solution of Eq.(3.18) is given by an arbitrary linear superposition of the $f_{i}$ defined in Eq.(3.19). Each of these functions behaves like $(1-z)^{-1}$ as $z \rightarrow 1$. However, Nørlund[16] showed that the difference between any two of the solutions Eq. (3.19) is regular at $z=1$. We require this regularity because $z=1$ corresponds to $x=0$, and the infra-red condition imposes finiteness of $f(1)$. We choose as two regular solutions the following:

$$
\begin{aligned}
& f_{1,3}(z)=f_{3}(z)-f_{1}(z) \\
& f_{2,3}(z)=f_{3}(z)-f_{2}(z) .
\end{aligned}
$$

These solutions, together with any one of Eq.(3.19), also form a fundamental system. Thus the most general solution can be written in the form

$$
f(z)=c_{1} f_{1,3}(z)+c_{2} f_{2,3}(z)+c_{3} f_{3}(z) .
$$

The infra-red condition immediately gives $c_{3}=0$, and the ultra-violet conditions lead to a system of linear homogeneous equations for the constants $c_{1}$ and $c_{2}$. In order for there to be a nontrivial solution of these homogeneous equations, the corresponding determinant must vanish, and one obtains in this way the ratio $c_{1} / c_{2}$. The undetermined common constant is fixed by the normalization $f(1)=1$. This vanishing of the determinant yields an equation for the dynamical mass; considering the large $\Lambda$ limit, we find, after some algebra,

$$
\begin{aligned}
\left(\alpha_{2}-\alpha_{3}\right)\left(\alpha_{2}+\alpha_{3}-1\right) \alpha_{1} & \prod_{i=1}^{3} \frac{\Gamma\left(\alpha_{2}+\gamma_{i}\right) \Gamma\left(\alpha_{3}+\gamma_{i}\right)}{\Gamma\left(\alpha_{2}-\alpha_{i}+1\right) \Gamma\left(\alpha_{3}-\alpha_{i}+1\right)}\left(\frac{\Lambda^{2}}{m^{2}}\right)^{-\alpha_{2}-\alpha_{3}} \\
& + \text { two cyclic permutations of }\{1,2,3\}=0 .
\end{aligned}
$$

In the case that the $\alpha$ 's are real, and with the ordering $\alpha_{3} \leq \alpha_{2} \leq \alpha_{1}$, the term written explicitly in Eq.(3.21) is the leading one, and in this case there 
is no nontrivial solution of Eq.(3.21), and hence none of Eq.(2.7). However, on increasing $\lambda$ (or equivalently the coupling $\alpha_{0}$ ), two of the roots $\left(\alpha_{1}\right.$ and $\alpha_{2}$ with our ordering) become equal, and then complex conjugate. For a cubic equation, it is easy to ascertain when this happens by looking at Cardano's formulas:

$$
\alpha_{3}=A+B-\frac{\lambda}{3}, \quad \alpha_{1}, \alpha_{2}=-\frac{A+B}{2} \pm \frac{\sqrt{3}}{2} i(A-B)-\frac{\lambda}{3},
$$

where

$$
\begin{gathered}
A=\left(-\frac{q}{2}+\sqrt{Q}\right)^{\frac{1}{3}}, \quad B=\left(-\frac{q}{2}-\sqrt{Q}\right)^{\frac{1}{3}}, \quad Q=\left(\frac{p}{3}\right)^{3}+\left(\frac{q}{2}\right)^{2}, \\
p=-\frac{1}{3} \lambda^{2}+\lambda-1, \quad q=\frac{2}{27} \lambda^{3}-\frac{1}{3} \lambda^{2}+\frac{4}{3} \lambda .
\end{gathered}
$$

When $Q<0$, Eq. 3.20 ) has three different real roots; but if $Q>0$ there are one real root and two complex conjugate ones. In the latter case two terms in Eq.(3.21) are of the same order, and then the equation for the dynamical mass can be written in the form

$$
\begin{aligned}
& \alpha_{2}\left(\alpha_{3}-\alpha_{1}\right)\left(\alpha_{3}+\alpha_{1}-1\right) \prod_{i=1}^{3} \frac{\Gamma\left(\alpha_{1}+\gamma_{i}\right)}{\Gamma\left(\alpha_{1}-\alpha_{i}+1\right)}\left(\frac{\Lambda^{2}}{m^{2}}\right)^{-\alpha_{1}} \\
& -\alpha_{1}\left(\alpha_{3}-\alpha_{2}\right)\left(\alpha_{3}+\alpha_{2}-1\right) \prod_{i=1}^{3} \frac{\Gamma\left(\alpha_{2}+\gamma_{i}\right)}{\Gamma\left(\alpha_{2}-\alpha_{i}+1\right)}\left(\frac{\Lambda^{2}}{m^{2}}\right)^{-\alpha_{2}}=0 .
\end{aligned}
$$

Writing $\alpha_{1}=u+i v$ and $\alpha_{2}=u-i v$, we obtain

$$
\sin \left[v \log \frac{\Lambda^{2}}{m^{2}}-\delta(\lambda)\right]=0
$$

where the phase is given by

$$
\delta(\lambda)=\arg \left[\alpha_{2}\left(\alpha_{3}-\alpha_{1}\right)\left(\alpha_{3}+\alpha_{1}-1\right) \prod_{i=1}^{3} \frac{\Gamma\left(\alpha_{1}+\gamma_{i}\right)}{\Gamma\left(\alpha_{1}-\alpha_{i}+1\right)}\right],
$$

and the mass is

$$
m=\Lambda \exp \left[-\frac{\pi n+\delta(\lambda)}{2 v}\right], \quad n=1,2, \ldots .
$$


As usual, the value $n=1$ corresponds to the ground state of the system. The critical coupling constant $\lambda_{c}\left(=\frac{3 \alpha_{c}}{4 \pi}\right)$, separating the chirally symmetric phase from that of spontaneous chiral symmetry breaking, is determined by the characteristic equation $Q=0$, i.e.

$$
3 \lambda^{4}-12 \lambda^{3}+32 \lambda^{2}+12 \lambda-4=0 .
$$

Numerical calculations give $\lambda_{c}=0.2172$ and

$$
\alpha_{1}\left(\lambda_{c}\right)=\alpha_{2}\left(\lambda_{c}\right) \equiv u_{c}=0.4435, \quad \alpha_{3}=-\lambda_{c}-2 u_{c}=-1.1042 .
$$

The above value of $\lambda_{c}$ implies $\alpha_{c}=0.9098$.

Near the critical value $\lambda \sim \lambda_{c}$, the imaginary part of the roots behaves like

$$
v(\lambda) \sim \sqrt{\lambda-\lambda_{c}}
$$

while $\delta(\lambda) \sim v$. Finally we obtain an expression for the dynamical mass:

$$
m=\Lambda \exp \left[-\frac{\sigma_{1}}{\sqrt{\lambda-\lambda_{c}}}+\sigma_{2}\right],
$$

where the constants $\sigma_{i}$ are given by

$$
\begin{aligned}
\sigma_{1}=- & \frac{\pi}{2} \frac{p\left(\lambda_{c}\right)}{\left[3 Q^{\prime}\left(\lambda_{c}\right)\right]^{\frac{1}{2}}} \\
\sigma_{2}=\frac{1}{2}\left[\frac{1}{u_{c}}+\frac{1}{1+\lambda_{c}+u_{c}}-2 \gamma\right. & \left.+\psi\left(\lambda_{c}+3 u_{c}\right)-\psi\left(1+\lambda_{c}+u_{c}\right)-\psi\left(2+u_{c}\right)-\psi\left(u_{c}\right)\right] .
\end{aligned}
$$

Here $\psi(x)=d \log \Gamma(x) / d x, \gamma$ is Euler's constant. Numerically, we find

$$
\sigma_{1}=1.525 \quad \sigma_{2}=1.604 .
$$




\subsection{Fourth-order Equation}

Our third approximation to the Curtis-Pennington contribution leads to the following fourth-order differential equation:

$$
\frac{\lambda}{2} \phi(x) f^{\prime \prime \prime \prime}+\left[1+\lambda \frac{x}{x+1}\right] x f^{\prime \prime \prime}+\left[3-\frac{\lambda}{2} \frac{x^{2}}{(x+1)^{2}}\right] f^{\prime \prime}+\frac{\lambda}{(x+1)^{2}}\left[f^{\prime}-f\right]=0,
$$

where

$$
\phi(x)=\int_{0}^{x} d y \frac{y^{2}}{y+1}=\frac{1}{2} x^{2}-x+\log (x+1) \underset{x>>1}{\sim} \frac{1}{2} x^{2} .
$$

Substituting $f \sim x^{-\alpha}$, we obtain the characteristic equation

$$
\lambda \alpha^{4}-2(2-\lambda) \alpha^{3}-3 \lambda \alpha^{2}+4(1-\lambda) \alpha-4 \lambda=0,
$$

with the critical value $\lambda_{c}=0.23320$ and the roots

$$
\begin{gathered}
\alpha_{1}=\alpha_{2}=0.4851 \\
\alpha_{3}=-1.1113 \\
\alpha_{4}=15.294 .
\end{gathered}
$$

These $\alpha$ 's and also $\sigma_{1}$ can be calculated using the explicit form for the roots of Eq.(3.28). 


\section{Chapter 4}

\section{Discussion}

A comparison of the results in the third- and fourth-order cases with those in the second-order case [Eq.(3.8)] shows that the numerical changes are rather small (recall that $\left.\alpha_{c}=\frac{4 \pi \lambda_{c}}{3}\right)$ :

\begin{tabular}{||c||c|c|c|c||}
\hline & $\alpha_{c}$ & $u_{c}$ & $\sigma_{1}$ & $\sigma_{2}$ \\
\hline 2nd order & 1.047 & 0.5 & 1.571 & 1.386 \\
3rd order & 0.910 & 0.443 & 1.525 & 1.604 \\
4th order & 0.980 & 0.485 & 1.462 & \\
\hline
\end{tabular}

We propose to show now that the form Eq.(3.23) is generic; that is, at any order of the quenched approximation, the same asymptotic formula is valid, with suitable values of the parameters $\lambda_{c}, \sigma_{1}$, and $\sigma_{2}$. Holdom's [14] essentially diagrammatic analysis, within the quenched approximation, leads to a bifurcation equation for the mass function of the following form

$$
f(x)=\lambda \int_{0}^{\frac{\Lambda^{2}}{m^{2}}} d y \frac{y f(y)}{y+1} K[x, y ; 0],
$$

where the kernel $K[x, y ; 0]$ is symmetric under $x \leftrightarrow y$ and $x K[x, y ; 0]$ is a function of $x / y$ only. This is known to be in agreement with a perturbative

\footnotetext{
${ }^{1}$ These numbers for $\alpha_{c}$ are in agreement with very recent numerical results of Curtis and Pennington 17
} 
expansion to order $O\left(\alpha^{2}\right)$ [18, and it leads immediately to a dynamical mass of the form Eq.(3.23).

Here we have shown that the same result also holds when we use a nonperturbative Ansatz for the vertex based on the Ward identity and multiplicative renormalizability, although this vertex does not lead to a bifurcation equation of the form Eq.(4.1). In general this result will hold for all approximation schemes in which the integral equation for the mass can be reduced to a differential equation of the Fuchsian type.

Once we have a differential equation, possibly of very high order, it is easy to understand that the behaviour of the mass function at large momenta is $x^{-\alpha}$, where $\alpha$ is a root of an (algebraic) characteristic equation of finite order (say $n$ ), with real coefficients. The onset of oscillations corresponds to equality of two of the roots of this characteristic equation: for larger values of the coupling, the two roots are complex conjugate and oscillatory behaviour is manifested and the ultra-violet conditions can be satisfied. To calculate the imaginary part $v$ of these roots, let us consider the characteristic equation's discriminant:

$$
\Delta=a_{0}^{2 n-2} \prod_{1 \leq i<j \leq n}\left(\alpha_{i}-\alpha_{j}\right)^{2}
$$

( $a_{0}$ is the coefficient of the largest power, $\alpha^{n}$ ). The discriminant is symmetric with respect to the roots $\alpha_{i}$, and it may be expressed in terms of the coefficients of the characteristic equation (for the rules to calculate the discriminant, see for example [19]). Thus $\Delta$ is an explicit function of $\lambda$. Note that $\Delta$ changes sign when a pair of complex conjugate roots appears.

The critical coupling constant $\lambda_{c}$ is determined by the condition

$$
\Delta\left(\lambda_{c}\right)=0
$$

and the imaginary part $v$ vanishes like $\Delta^{\frac{1}{2}}$. Normally $\lambda_{c}$ is a simple root of Eq.(4.2), so that

$$
v \sim \sqrt{-\Delta^{\prime}\left(\lambda_{c}\right)} \sqrt{\lambda-\lambda_{c}}
$$

and this leads to formula Eq.(3.23) for the dynamical mass. In principle $\lambda_{c}$ could be a degenerate root, but this would be an 'accident': the generic case is as just given. 
Our considerations show that the dependence of the dynamical mass on the coupling constant, Eq. 3.23), is a universal feature of quenched theories. The behaviour of the real part of those roots, $u$, is however not universal. It gives the asymptotic power-envelope of the decreasing and oscillating mass function:

$$
m\left(p^{2}\right) \sim\left[\frac{1}{p^{2}}\right]^{u}=\left[\frac{1}{p}\right]^{2-\gamma_{m}},
$$

where $\gamma_{m}$ is the anomalous dimension of the composite operator $\bar{\psi} \psi$. In the lowest approximation (the second-order equation), we have $\gamma_{m}=1$, but the Curtis-Pennington Ansatz leads to a modification of this dimension. For example, in the cubic approximation we find $\gamma_{m}=1.114$. Values $\gamma_{m} \geq 1$ imply that some four-fermion operators like $(\bar{\psi} \psi)^{2},\left(\bar{\psi} i \gamma_{5} \psi\right)^{2}$, become relevant in the continuum limit and must be added to the QED Lagrangian [4], which means that pure QED is not self-consistent.

\section{Acknowledgements}

We would like to thank V.A. Miransky, M.R. Pennington and F. Wagener for useful discussions. Two of us (VPG and PM) wish to thank the Stichting FOM (Fundamenteel Onderzoek der Materie), financially supported by the Nederlandse Organisatie voor Wetenschappelijk Onderzoek, for its support. 


\section{Bibliography}

[1] T. Maskawa and H. Nakajima, Prog. Theor. Phys. 52 (1974) 1326.

[2] P.I. Fomin, V.P. Gusynin, V.A. Miransky and Yu.A. Sitenko, Riv. Nuovo Cim. 6 (1983) 1.

[3] P.I. Fomin, V.P. Gusynin and V.A. Miransky, Phys. Lett. B78 (1978) 136; V.A. Miransky, Nuovo Cim. 90A (1985) 149.

[4] W.A. Bardeen, C. Leung and S. Love, Phys. Rev. Lett. 56 (1986) 1230; Nucl. Phys. B273 (1986) 649.

[5] D.C. Curtis and M.R. Pennington, Phys. Rev. D42 (1990) 4165; D46 (1992) 2663.

[6] J. Oliensis and P.W. Johnson, Phys. Rev. D42 (1990) 656; V. Gusynin, Mod. Phys. Lett. A5 (1990)133; K. Kondo and H. Nakatani, Nucl. Phys. B351 (1990) 236.

[7] D. Atkinson and D.W.E. Blatt, Nucl. Phys. B151 (1979) 342.

[8] G.H. Pimbley, "Eigenfunction branches of nonlinear operators, and their bifurcations", Lecture Notes in Mathematics, Vol. 104 (Springer, Berlin, 1969); see also D. Atkinson, J. Math. Phys. 28 (1987) 2494.

[9] V.A. Miransky, Phys. Lett. B165 (1985) 401; V.P. Gusynin and V.A. Miransky, Phys. Lett. B191 (1987) 141.

[10] D. Atkinson and P.W. Johnson, Phys. Rev. D35 (1987) 1943. 
[11] J. Kogut, E. Dagotto and A. Kocić, Phys. Rev. Lett. 59 (1988) 772; Nucl. Phys. B317 (1989) 253, 271; Phys. Rev. D43 (1991) R1763.

[12] K. Lane, Phys. Rev. D10 (1974) 2605; H. Politzer, Nucl. Phys. B 117(1976) 397; V.A. Miransky, Sov. J. Nucl. Phys. 38 (1984) 280; K. Higashijima, Phys. Rev. D29 (1984) 1228.

[13] T. Akiba and T. Yanagida, Phys. Lett. B169 432; K. Yamawaki, M. Bando and K. Matumoto, Phys. Rev. Lett. 56 (1986) 1335; M. Bando, T. Morozumi, H. So and K. Yamawaki, Phys. Rev. Lett. 59 (1987) 389.

[14] B. Holdom, Phys. Rev. Lett. 62 (1989) 997.

[15] U. Mahanta, Phys. Lett. B225 (1989) 187.

[16] N.E. Nørlund, "Hypergeometric Functions", Acta Mathematica 94 (1955) 289.

[17] Private communication from M.R. Pennington.

[18] T. Appelquist, K. Lane and U. Mahanta, Phys. Rev. Lett. 61 (1988) 1553.

[19] G.A. Korn and T.M. Korn, "Mathematical Handbook for Scientists and Engineers", McGraw-Hill (1968). 